\title{
Valore diagnostico del profilo urinario steroideo nella valutazione dei tumori surrenalici
}

\author{
Emanuela Arvat ${ }^{1}$
}

Pubblicato online: 20 ottobre 2015

(C) Springer International Publishing AG 2015

Commento a:

Diagnostic value of urinary steroid profiling in the evaluation of adrenal tumors.

T.M. Kerkhofs, M.N. Kerstens, I.P. Kema, T.P. Willems,

H.R. Haak.

Horm Cancer (2015) 6:168-175

Le masse surrenaliche di riscontro occasionale rappresentano un'entità clinica in costante aumento, con una prevalenza che raggiunge il $5 \%$ al riscontro TC nella popolazione anziana. I punti critici della diagnosi risiedono nell'identificazione degli stati iperfunzionanti e nella diagnosi differenziale fra masse benigne e maligne. La prevalenza, infatti, del carcinoma nell'ambito dei tumori del surrene varia dall'1 al 12\%. Il sospetto di malignità si fonda perlopiù sulle caratteristiche radiologiche (dimensioni, disomogeneità, necrosi, calcificazioni, densità valutata in termini di Unità Hounsfield). Anche il follow-up delle masse dubbie e non operate si basa sull'esecuzione periodica dell'imaging, più frequentemente la TC, indagine non esente da rischi oncologici a lungo termine. Sebbene l'ipersecrezione steroidea non sia ad oggi considerata un parametro validato come indice di malignità, recenti evidenze indicano come i carcinomi surrenalici presentino un particolare profilo secretorio, differente dalle altre masse surrenaliche.

Questo studio ha valutato, in maniera retrospettiva, il profilo urinario di 18 steroidi surrenalici in 152 pazienti con tumore di riscontro occasionale (di cui 27 con carcinoma, ACC), valutati con cromatografia gassosa associata a spettrometria di massa. I pazienti con ACC hanno presentato

$\triangle$ E. Arvat

emanuela.arvat@unito.it

1 Divisione di Endocrinologia Oncologica, Dipartimento di Scienze Mediche, Università di Torino, Torino, Italia un profilo di aumentata secrezione rispetto a tutte le altre masse, in particolare a carico di alcuni steroidi (tetraidro11-desossicortisolo, THS; pregnanediolo, P2; pregnanetriolo, P3; etiocolanolone, E; androsterone: tetraidrocortisolo, THF; e tetraidrocortisone, THE). La curva ROC per il THS ha evidenziato una sensibilità del $100 \%$ e una specificità del $99 \%$ con un cut-off di 2,35 micromol/24 h. È stata anche evidenziata una correlazione fra diametro dell' ACC e livelli urinari di THS. Non sono invece state dimostrate differenze significative nel profilo secretorio per quanto riguarda $\mathrm{i}$ tumori non carcinomatosi. Non è chiara l'eziologia dell'ipersecrezione di tali steroidi a carico del tessuto carcinomatoso: sono stati ipotizzati sia un accumulo di precursori per eccesso di attività funzionale sia disturbi a carico degli enzimi coinvolti nella cascata steroidogenetica della corteccia surrenalica.

Lo studio, in accordo con quanto precedentemente segnalato in letteratura, dimostra la potenziale utilità del profilo urinario di alcuni steroidi surrenalici nella diagnosi di carcinoma del surrene, anche in assenza di sintomatologia clinica correlata a iperfunzione ormonale. Inoltre, tale metodica potrebbe rivelarsi utile anche nel follow-up delle masse non operate. Tale valutazione, qualora eseguita in laboratori centralizzati con disponibilità della metodica cromatografica, potrebbe essere meno costosa e meno gravata da potenziali effetti negativi rispetto all'imaging, in particolare se utilizzata nel follow-up. Tuttavia, solo studi multicentrici disegnati su ampie popolazioni di pazienti saranno in grado di validare o meno questa metodica nei pazienti con tumore surrenalico. 